\title{
Effect of time of day and fasting duration on measures of glycaemia: analysis from the Whitehall II Study
}

\author{
A. Hulmán • K. Færch • D. Vistisen • J. Karsai • \\ T. A. Nyári • A. G. Tabák • E. J. Brunner • \\ M. Kivimäki • D. R. Witte
}

Received: 30 July 2012 / Accepted: 18 October 2012 /Published online: 10 November 2012

(C) Springer-Verlag Berlin Heidelberg 2012

\begin{abstract}
Aims/hypothesis We aimed to study diurnal variation in glucose regulation by examining the effects of time of day and fasting duration on fasting plasma glucose (FPG), $2 \mathrm{~h}$ post-load plasma glucose (2hPG) and $\mathrm{HbA}_{1 \mathrm{c}}$ levels.

Methods We analysed data from 5,978 non-diabetic white men and women from the prospective Whitehall II Study. All studied participants fasted for at least $8 \mathrm{~h}$ before a clinical examination, which included an OGTT and anthropometric measurements. We fitted mixed-effects models for FPG, $2 \mathrm{hPG}$ and $\mathrm{HbA}_{1 \mathrm{c}}$ as outcome variables, and time of day and/or fasting duration as explanatory variables. Models were adjusted for age, BMI and study phase.
\end{abstract}

Electronic supplementary material The online version of this article (doi:10.1007/s00125-012-2770-3) contains peer-reviewed but unedited supplementary material, which is available to authorised users.

A. Hulmán $(\bowtie) \cdot J$. Karsai $\cdot$ T. A. Nyári

Department of Medical Physics and Medical Informatics,

University of Szeged,

Korányi fasor 9 ,

H-6720, Szeged, Hungary

e-mail: hulman.adam@med.u-szeged.hu

K. Færch $\cdot$ D. Vistisen $\cdot$ D. R. Witte

Steno Diabetes Center A/S,

Gentofte, Denmark

A. G. Tabák · E. J. Brunner • M. Kivimäki

Department of Epidemiology and Public Health,

University College London,

London, UK

A. G. Tabák

First Department of Medicine,

Semmelweis University Faculty of Medicine,

Budapest, Hungary

D. R. Witte

Centre de Recherche Public de la Santé,

Strassen, Luxembourg
Results Time of day and fasting duration were associated inversely with FPG and positively with $2 \mathrm{hPG}$. The mean difference between measures at 08:00 and 15:00 hours in $\mathrm{men} /$ women was $-0.46(95 \% \mathrm{CI}-0.50,-0.42) \mathrm{mmol} / \mathrm{l} /-0.39$ $(95 \% \mathrm{CI}-0.46,-0.31) \mathrm{mmol} / \mathrm{l}$ and 1.39 (95\% CI $1.25,1.52)$ $\mathrm{mmol} / \mathrm{l} / 1.19(95 \% \mathrm{CI} 0.96,1.42) \mathrm{mmol} / \mathrm{l}$ for $\mathrm{FPG}$ and $2 \mathrm{hPG}$, respectively. $\mathrm{HbA}_{1 \mathrm{c}}$ levels were independent of either time. Time of day and fasting duration were independently associated with 2 hPG. In contrast, the effect of fasting duration on FPG was markedly attenuated with adjustment for time of day. Ageing, but not obesity, was associated with increased diurnal variation in glucose tolerance.

Conclusions/interpretation Both time of day and fasting duration should be considered in clinical practice and epidemiological studies, since they have clinically relevant effects on FPG and $2 \mathrm{hPG}$ levels. As biochemically expected, $\mathrm{HbA}_{1 \mathrm{c}}$ levels are independent of time of blood sampling and fasting duration.

Keywords Diurnal variation $\cdot$ Fasting duration $\cdot \mathrm{HbA}_{1 \mathrm{c}}$. Oral glucose tolerance test

Abbreviations
$\begin{array}{ll}2 \mathrm{hPG} & 2 \mathrm{~h} \text { post-load plasma glucose } \\ \text { FPG } & \text { Fasting plasma glucose } \\ \text { IQR } & \text { Interquartile range }\end{array}$

Introduction

Diurnal variation in glucose tolerance was described more than 40 years ago [1-3]. Nevertheless, it is still not taken into account sufficiently in clinical practice or in epidemiological studies performing OGTTs. More recent studies have focused on the effect of either time of day or fasting duration on fasting plasma glucose (FPG) [4-6], and to a 
lesser extent on post-load plasma glucose [7]. However, their joint effect, especially on $2 \mathrm{~h}$ post-load plasma glucose $(2 \mathrm{hPG})$, has never been analysed comprehensively.

Recently, $\mathrm{HbA}_{1 \mathrm{c}}$ was included among the diagnostic tools for diabetes. Although it is biochemically fully expected that $\mathrm{HbA}_{1 \mathrm{c}}$ is not affected by time of day or fasting duration, no previous studies have confirmed this at a population level. Therefore we aimed to analyse the individual and joint effect of time of day and fasting duration on FPG, $2 \mathrm{hPG}$ and $\mathrm{HbA}_{1 \mathrm{c}}$ and whether these associations are affected by ageing and obesity.

\section{Methods}

We analysed data from the Whitehall II cohort. In 1985, 10,308 British civil servants were recruited from offices in London (phase 1). The participants were invited to subsequent phases with a questionnaire every 2.5 years and a clinical examination every 5 years. OGTTs with measurements of FPG and $2 \mathrm{hPG}$ were first performed in phase 3 (1991-1993; 6,712 participants of white ethnicity with valid glucose measures). $\mathrm{HbA}_{1 \mathrm{c}}$ was measured only in phases 7 (2002-2004) and 9 (2007-2009). Time of fasting blood draw was recorded by the research nurse who manned the OGTT station as HH:MM on the participant's clinical report form based on a standardised clock in the examination room. Blood samples were handled according to standard protocols. The University College London ethics committee reviewed and approved the study. Written informed consent was obtained from all participants at each study phase. The Whitehall II Study is described in detail elsewhere [8].

We excluded from the main analysis examinations of participants with diabetes or on glucose-lowering treatment $(n=1,266)$. Further examinations were excluded because of unrealistic fasting duration (last meal before 17:00 hours on the previous day or after 01:00 hours on the day of the examination; $n=334$ ) or missing contemporary weight or height measures $(n=407)$. The final dataset included 5,978 participants (with 13,269 personexaminations over an 18-year period) of white ethnicity, aged from 40 to 80 years, in whom FPG measures were taken between 08:00 and 15:00 hours (median 10:42, interquartile range [IQR] 9:50-11:36) and fasting duration ranged from 8 to $20 \mathrm{~h}$ (median 13.4, IQR 12.1-14.9).

The impact of time of day and fasting duration on FPG, 2hPG and $\mathrm{HbA}_{1 \mathrm{c}}$ was assessed in age- and BMIadjusted mixed-effects models stratified by sex. Models for $2 \mathrm{hPG}$ were also adjusted for height, as previously suggested [9]. To handle the within-person correlation arising from the longitudinal structure of the dataset, random effects were included for the intercept in each model. We also fitted the described models with standardised outcome variables in order to compare the relative impact of time of day and fasting duration on the different glucose measures. After the individual effect of time of day and fasting duration had been examined, all models were fitted with both variables included at the same time. Time of day and fasting duration were moderately related (Pearson $r=0.6, p<0.001$ ). The effect of ageing and BMI on diurnal variation was investigated by including interaction terms in the models. Statistical analysis was performed using $\mathrm{R}$ version 2.15.1. Statistical significance was inferred at a 5\% level.

\section{Results}

The results from mixed-effects models with $\mathrm{FPG}, 2 \mathrm{hPG}$ and $\mathrm{HbA}_{1 \mathrm{c}}$ as outcome variables and time of day and/or fasting duration as explanatory variables are presented in Table 1.

FPG levels were inversely associated with time of day and fasting duration (e.g. in men $-0.46 \mathrm{mmol} / 1$ (95\% CI $-0.50,-0.42$ ) mean difference in FPG between measures at 08:00 and 15:00 hours). After inclusion of both variables in the same model, the effect of fasting duration on FPG was markedly attenuated in both men and women.

Time of day and fasting duration were positively associated with $2 \mathrm{hPG}$ in both men and women (e.g. in men $1.39 \mathrm{mmol} / 1$ (95\% CI 1.25, 1.52) mean difference in 2hPG between measures at 08:00 and 15:00 hours). After inclusion of both variables in the model, the effect of each remained statistically significant. Adding time of day to the model including fasting duration improved the model fit more than adding fasting duration to the model including time of day.

We investigated the potential effect of our findings on the diagnosis of diabetes by modelling the hypothetical situation that all OGTTs started exactly at 09:00 hours. We found that, in this scenario, 36 (14.6\%) people with WHO-defined diabetes would not receive a diabetes diagnosis, and seven people without diabetes would be classified as having diabetes.

Diurnal variation in $2 \mathrm{hPG}$ increased with age (electronic supplementary material [ESM] Table 1), but this was not the case for FPG. Higher BMI was associated with greater diurnal variation in FPG (ESM Table 2). No interaction of BMI and time of day for $2 \mathrm{hPG}$ was observed.

$\mathrm{HbA}_{1 \mathrm{c}}$ levels were not associated with time of day or fasting duration.

Models with standardised outcome variables showed that the relative impact of time of day and fasting duration were of similar magnitude for FPG and 2hPG, but in the opposite direction (Fig. 1). 
Table 1 Estimated coefficients of time of day (TD) and fasting duration (FD) from age- and BMI-adjusted mixed-effects models for FPG, $2 \mathrm{hPG}$ and $\mathrm{HbA}_{1 \mathrm{c}}$, with $95 \% \mathrm{CIs}$

All models were also adjusted for study phase

$* p<0.05, * * * p<0.001$

\begin{tabular}{lll}
\hline Model variables & Men & Women \\
\hline FPG (mmol/l) & $-0.066(-0.072,-0.059)^{* * *}$ & $-0.055(-0.066,-0.044)^{* * *}$ \\
Only TD (h) & $-0.033(-0.037,-0.028)^{* * *}$ & $-0.024(-0.031,-0.017)^{* * *}$ \\
Only FD (h) & & \\
TD and FD & $-0.059(-0.067,-0.050)^{* * *}$ & $-0.053(-0.067,-0.039)^{* * *}$ \\
TD (h) & $-0.007(-0.012,-0.001)^{*}$ & $-0.002(-0.011,0.007)$ \\
FD (h) & $0.198(0.179,0.217)^{* * *}$ & \\
2hPG (mmol/l) & $0.124(0.111,0.137)^{* * *}$ & $0.170(0.137,0.203)^{* * *}$ \\
Only TD (h) & $0.128(0.102,0.154)^{* * *}$ & $0.103(0.083,0.124)^{* * *}$ \\
Only FD (h) & $0.068(0.051,0.085)^{* * *}$ & $0.111(0.068,0.154)^{* * *}$ \\
TD and FD & & $0.058(0.030,0.085)^{* * *}$ \\
TD (h) & $0.000(-0.007,0.007)$ & \\
FD (h) & $0.000(-0.004,0.005)$ & $-0.006(-0.018,0.006)$ \\
HbA $(\%)$ & $-0.001(-0.009,0.008)$ & $-0.003(-0.011,0.005)$ \\
Only TD (h) & $0.001(-0.005,0.006)$ & $-0.005(-0.021,0.011)$ \\
Only FD (h) & & $-0.001(-0.012,0.009)$ \\
TD and FD & & \\
TD (h) & FD (h) &
\end{tabular}

\section{Discussion}

In a large occupational cohort study, we showed that time of blood sampling and duration of fasting before blood sampling affect FPG and 2hPG levels considerably. As expected, neither of these factors affected $\mathrm{HbA}_{1 \mathrm{c}}$. Furthermore, we showed how age and BMI affect the diurnal variation of glucose measures.

Fasting glucose The observed decline in FPG levels during the day is in line with previous findings, which also showed clinically relevant differences between morning and early afternoon FPG measures [4]. Our findings support the recommendation that standardisation of fasting duration and time of day is important in epidemiological studies and that adjustment for these factors is advisable where the necessary data are available [5]. These results indicate that timing should not be ignored when diagnosing diabetes, or comparing individual glucose levels longitudinally in clinical practice. Our results are consistent with previous findings on the modest role of fasting duration beyond $3 \mathrm{~h}$ in addition to time of day when measuring FPG and that more effort on the standardisation or assessment of other factors, such as diet and physical activity, is necessary [6].

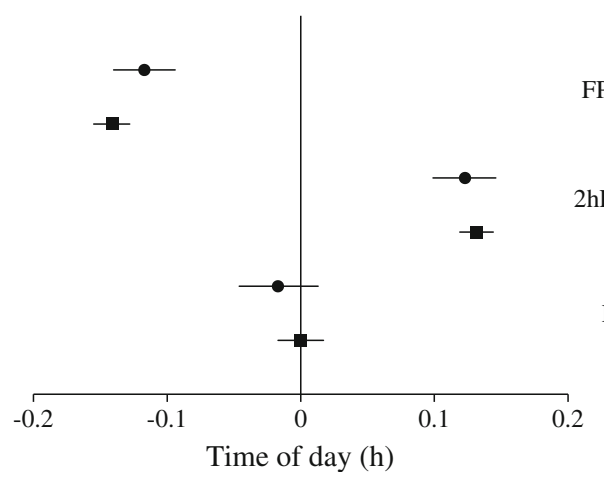

Fig. 1 Standardised estimated coefficients of time of day and fasting duration are presented for men (black squares) and women (black circles) with $95 \%$ CIs. The displayed values are the differences caused by an hour difference in time of day and fasting duration given in SD

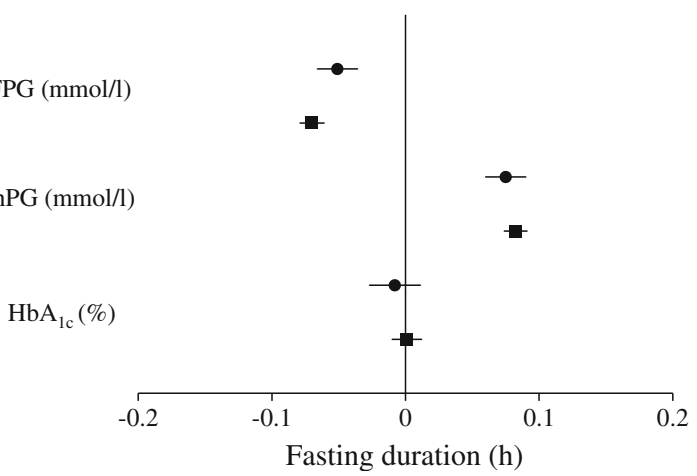

for each variable (1 SD is 0.47 and $0.47 \mathrm{mmol} / 1$ for $\mathrm{FPG}, 1.51$ and $1.39 \mathrm{mmol} / \mathrm{l}$ for $2 \mathrm{hPG}$, and $0.41 \%$ and $0.42 \%$ for $\mathrm{HbA}_{1 \mathrm{c}}$ for men and women, respectively) 
Glucose tolerance Our results are consistent with previous evidence indicating higher $2 \mathrm{hPG}$ levels in the afternoon than in the morning [1-3] and that diurnal variation in glucose tolerance increases with age. However, we found no unanimous evidence to suggest that obesity would reduce diurnal variation in $2 \mathrm{hPG}$ [2]. A previous review reported that there is no evidence for an effect of fasting duration in addition to time of day [10]. In contrast, we show that for $2 \mathrm{hPG}$, fasting duration improved the model fit and was statistically highly significant, even if time of day was accounted for. Possible reasons for this difference could include differences in study settings (in most of the previous studies, people who were examined in the afternoon were allowed a full breakfast) and statistical power. Although the studies are not directly comparable, our results support the recent finding of an underestimation of glucose tolerance among pregnant women who had an afternoon rather than a morning $1 \mathrm{~h}, 50 \mathrm{~g}$, non-fasting glucose challenge test, as compared with a confirmatory morning $3 \mathrm{~h}, 100 \mathrm{~g}$ fasting OGTT performed on a separate day [7].

$H b A_{1 c}$ Using a relatively large sample, we confirmed that $\mathrm{HbA}_{1 \mathrm{c}}$ levels are affected by neither time of day nor fasting duration. This finding highlights a key strength of $\mathrm{HbA}_{1 \mathrm{c}}$ as a diabetes diagnostic measure.

Diurnal variation in glucose tolerance has not received much attention in the past decades, even though the definition of diabetes was based on fasting and/or $2 \mathrm{~h}$ post-OGTT values. Since the introduction of $\mathrm{HbA}_{1 \mathrm{c}}$ as an additional diagnostic criterion, many analyses, which have shown a modest degree of overlap between $\mathrm{HbA}_{1 c^{-}}$and OGTT-based diabetes diagnosis, have implicitly assumed the OGTT to be a type of gold standard. Our analysis, based on a large sample of repeated measures in a well-characterised cohort, shows that even a few hours difference in fasting duration or sampling time can affect whether or not somebody is diagnosed as having diabetes based on an OGTT.

The key strength of our study is the detailed assessment of both time of day and fasting duration, which made it possible to include them as continuous variables in the models. However, the relatively low proportion of women in the study resulted in wider confidence intervals for their estimated coefficients. Further research is needed to examine the generalisability of our findings to other populations, ethnic groups and pregnant women.

In conclusion, our results show the importance of standardising the diagnostic OGTT regarding time of day and fasting duration, which is often overlooked in clinical practice and epidemiological investigations. 2hPG measures after an OGTT vary by different fasting duration periods even after $8 \mathrm{~h}$ of fasting, independently of the time of blood sampling. Timing effects should be taken into consideration to avoid biased comparisons or classifications based on FPG and $2 \mathrm{hPG}$ values.
Acknowledgements We thank all participants and members of the Whitehall II Study team.

Funding The Whitehall II Study was supported by the following organisations: Medical Research Council, UK, Economic and Social Research Council, UK, British Heart Foundation, UK, Health and Safety Executive, UK, Department of Health, UK, BUPA Foundation, UK, National Heart Lung and Blood Institute, USA and NIH: National Institute on Ageing, USA. The Steno Diabetes Center receives part of its core funding from unrestricted grants from the Novo Foundation and Novo Nordisk A/S. M. Kivimäki is supported by an ESRC professorship.

Duality of interest K. Færch, D. Vistisen and D. R. Witte are employed by the Steno Diabetes Center A/S, a research hospital working in the Danish National Health Service and owned by Novo Nordisk A/S. They also own shares in Novo Nordisk A/S.

Contribution statement $\mathrm{AH}, \mathrm{KF}$ and DV contributed to the study conception, analysis and interpretation of data, and drafting and revision of the manuscript. JK and TAN contributed to the statistical analysis, and reviewed and edited the manuscript. AGT, EJB and MK are Whitehall II Investigators, who contributed data and reviewed and edited the manuscript. DRW contributed to the study design and conception, interpretation of data, and drafting and revision of the manuscript. All authors approved the final version of the manuscript to be published.

\section{References}

1. Jarrett RJ, Keen H (1969) Diurnal variation of oral glucose tolerance: a possible pointer to the evolution of diabetes mellitus. BMJ 2:341-344

2. Zimmet PZ, Wall JR, Rome R, Stimmler L, Jarrett RJ (1974) Diurnal variation in glucose tolerance: associated changes in plasma insulin, growth hormone, and non-esterified fatty acids. BMJ 1:485-488

3. Mayer KH, Stamler J, Dyer A et al (1976) Epidemiologic findings on the relationship of time of day and time since last meal to glucose tolerance. Diabetes 25:936-943

4. Troisi RJ, Cowie CC, Harris MI (2000) Diurnal variation in fasting plasma glucose: implications for diagnosis of diabetes in patients examined in the afternoon. JAMA 284:3157-3159

5. Emberson JR, Whincup PH, Walker M, Thomas M, Alberti KGMM (2002) Biochemical measures in a population-based study: effect of fasting duration and time of day. Ann Clin Biochem 39:493-501

6. Moebus S, Göres L, Lösch C, Jöckel K-H (2011) Impact of time since last caloric intake on blood glucose levels. Eur J Epidemiol 26:719-728

7. Goldberg RJ, Ye C, Sermer M et al (2012) Circadian variation in the response to the glucose challenge test in pregnancy: implications for screening for gestational diabetes mellitus. Diabetes Care $35: 1578-1584$

8. Tabák AG, Jokela M, Akbaraly TN, Brunner EJ, Kivimäki M, Witte DR (2009) Trajectories of glycaemia, insulin sensitivity, and insulin secretion before diagnosis of type 2 diabetes: an analysis from the Whitehall II study. Lancet 373:2215-2221

9. Faerch K, Borch-Johnsen K, Vaag A, Jørgensen T, Witte DR (2010) Sex differences in glucose levels: a consequence of physiology or methodological convenience? The Inter99 study. Diabetologia 53:858-865

10. Van Cauter E, Polonsky KS, Scheen AJ (1997) Roles of circadian rhythmicity and sleep in human glucose regulation. Endocr Rev 18:716-738 